information about the aetiology of their deficiency, their symptoms, management and response to supplementation.

Methods We reviewed all Vitamin A assay requests which were reported as $<1 \mathrm{umol} / \mathrm{L}$ across a 5 year period between 2012-2016. We looked at the indication for the test, whether the patient was symptomatic, and what their symptoms were. In addition, we collected data regarding past medical history.

Results We identified 80 patients with Vitamin A deficiency. Of our cohort, 16 patients were symptomatic, presenting with predominantly visual symptoms: blurred vision, night blindness, recurrent miscarriage, poor vision and xerophthalmia. Vitamin A assays were requested by a variety of departments but most commonly by Liver, Gastroenterology/Nutrition and Ophthalmology. Only one of our symptomatic patient's Vitamin A deficiency was due primarily to poor intake and in one patient the aetiology of Vitamin A deficiency was unknown. Our other symptomatic patients had coexisting chronic illnesses which resulting in malabsorption.

Conclusion We demonstrated that Vitamin A deficiency is a cause of morbidity and potentially mortality in a developed country. We have also shown that the cause of deficiency in developed countries is not the same as in developing countries. We found the cause of Vitamin A deficiency to be overwhelmingly due to malabsorption, most commonly secondary to surgery or hepatobiliary disease.

\section{PWE-118 HYPOPHOSPHATEMIA FOLLOWING IRON INFUSION IN PATIENTS RECEIVING HOME PARENTERAL NUTRITION IN A REGIONAL NUTRITION CENTRE}

A Merghani Mohamed*, H Layland, NP Thompson, CG Mountford. Department of Gastroenterology, Freeman Hospital, Newcastle upon Tyne Hospitals NHS Foundation Trust

\subsection{6/gutjnl-2018-BSGAbstracts.352}

Background Hypophosphatemia is a recognised complication of iron infusion. Patients receiving Home Parenteral Nutrition (HPN) due to intestinal failure are at additional risk of hypophosphatemia due to their underlying disease state. This study aimed to identify the extent of hypophosphatemia following iron infusion in this group of patients.

Methods The medical records of all HPN patients treated with parenteral iron infusion in the Department of Gastroenterology, Freeman Hospital, between April 2012 and February 2017 were retrospectively reviewed. Patients were identified from the regional HPN electronic database.

Patient's demographics, type and dose of iron infusion were noted. Blood test parameters including haemoglobin, ferritin, transferrin saturations and phosphate levels were analysed before and after iron infusion. Management of abnormal phosphate level was documented.

Degree of hypophosphataemia was categorised as severe $(<0.30 \mathrm{mmol} / \mathrm{L})$, moderate $(0.30$ to $0.59 \mathrm{mmol} / \mathrm{L})$, mild $(0.59$ to $0.79 \mathrm{mmol} / \mathrm{L}$ ). Normal phosphate level was defined between 0.80 to $1.50 \mathrm{mmol} / \mathrm{L}$, elevated levels above $1.50 \mathrm{mmol} / \mathrm{L}$.

Results Thirty five patients (19 females, 16 males), mean age 54, received iron infusions. All patients received Ferrinject iron infusion (Vifor Pharma UK Limited) at a dose appropriate to manufacturer recommendations, based on bodyweight. Results demonstrated that phosphate levels fell in 7 out of 35 patients (20\%) following iron infusion; 2 with severe hypophosphataemia (5.7\%) and 5 with mild hypophosphataemia (14.3\%). Table 1 summarises the effect on phosphate level of iron infusion. Twenty seven out of 35 patients (71\%) had phosphate levels checked within 2 months post iron infusion.

One patient who developed severe hypophosphataemia had mild hypophosphataemia prior to iron infusion. All other patients had normal phosphate levels beforehand. Both patients with severe hypophosphataemia required intravenous phosphate replacement via manipulation of their HPN prescription. Of the 5 patients who developed mild hypophosphataemia, 4 resolved spontaneously, 1 required HPN prescription change. Time to normalisation of phosphate ranged from 4 weeks to 5 months.

Conclusions Severe Hypophosphatemia is a rare but potentially significant occurrence following iron infusion and can be prolonged in patients receiving Home Parenteral Nutrition. Numbers in this study are small and more studies are needed to investigate this further, including assessing the incidence using other preparations of intravenous iron in this group of patients. Routinely checking phosphate levels after iron infusions in this group of patients is probably warranted.

\section{PWE-119 IRON ISOMALTOSIDE TO IMPROVE OESOPHAGOGASTRIC ADENOCARCINOMA RELATED ANAEMIA AND QUALITY OF LIFE DURING CHEMOTHERAPY}

${ }^{1}$ Oliver $\mathrm{Ng},{ }^{1}$ Barrie Keeler, ${ }^{1} J o h n$ Alastair Simpson, ${ }^{1}$ Srinivasan Madhusudan, ${ }^{2}$ Matthew J Brookes*, 'Austin G Acheson. 'University Of Nottingham, Nottingham, UK; ${ }^{2}$ Royal Wolverhampton Hospital, Wolverhampton, UK

\subsection{6/gutjnl-2018-BSGAbstracts.353}

Introduction Anaemia is common in oesophagogastric (OG) adenocarcinoma, increasing mortality, blood transfusions and reducing quality of life with no clear evidence exists for safe and effective treatment, especially for mild to moderate anaemia. This study assessed the efficacy of intravenous iron isomaltoside to improve anaemia, quality of life and prevent blood transfusions in OG adenocarcinoma.

Methods Anaemic patients with histologically proven OG adenocarcinoma were recruited before initiation of palliative chemotherapy. Patients were randomised to receive standard care or intravenous iron isomaltoside. Post-chemotherapy changes in haemoglobin, ferritin, transferrin saturations, blood transfusions and quality of life were recorded for 3 cycles of chemotherapy.

Results 27 patients were randomised to standard care $(n=13)$ or intravenous iron $(n=14)$. A non-significant decrease in haemoglobin was seen in the standard care group over three cycles of chemotherapy (mean difference-0.6 g/dL 95\% CI-0.1 to-1.1 $\mathrm{g} / \mathrm{dL}, \mathrm{p}=0.336$ ) compared to an increase in the intravenous iron group (mean difference $0.5 \mathrm{~g} / \mathrm{dL} 95 \% \mathrm{CI}-0.1 \mathrm{~g} / \mathrm{dL}$ to $1.1 \mathrm{~g} / \mathrm{dL}, \mathrm{p}=0.903)$. An increase in ferritin and transferrin saturations above $20 \%$ was seen in the intravenous iron group by cycle one of chemotherapy with a greater and statistically significant increase in ferritin in the intravenous iron group (standard care $116 \mathrm{ng} / \mathrm{mL}$ versus intravenous iron group $770 \mathrm{ng} / \mathrm{mL}, \mathrm{p}<0.05)$.

Blood transfusions were received by 7 patients (standard care $n=4$, intravenous iron $n=3$ ). No significant difference in the number and amount of blood transfused were seen $(p=0.851)$. No patient received a blood transfusion after cycle one of chemotherapy in the intravenous iron group.

Quality of life improved in the intravenous iron group with physical well-being, emotional well-being, anaemia-specific 
quality of life, trial outcome index and total scores all exceeding the minimum clinically important difference. No improvement was seen with standard care.

Conclusions Data from this pilot study suggest intravenous iron improves quality of life, ferritin and transferrin saturations. It may also increase or maintain haemoglobin, thus preventing transfusions after the first cycle of chemotherapy. However, larger adequately powered studies are required to definitively conclude this.

\section{PWE-120 AN EXPERIENCE OF HOME TOTAL PARENTERAL NUTRITION AT THE FREEMAN HOSPITAL OVER A 21- YEAR PERIOD}

Satyasheel Ramful* ${ }^{*}$ Hayley Leyland, Colette Louise Kirk, Lisa Gemmell, Christopher G Mountford, Nicholas P Thompson. Depts of Gastroenterology and Dietetics, Freeman Hospital, Newcastle Upon Tyne, UK

\subsection{6/gutjnl-2018-BSGAbstracts.354}

Introduction Home total parenteral nutrition (HPN) is used for patients with severe intestinal failure. Our service at the Freeman Hospital, Newcastle upon Tyne covers Northumberland, North Cumbria, Durham, Gateshead, North Yorkshire, Tyneside, Wearside and Teesside.

Methods This study used the database kept by the HPN service and the Trust document portal to gather data retrospectively regarding 221 patients started on HPN from 1996 till 2016.25 individual data sets were collated about each patient. Line sepsis rates were obtained from the trust central venous catheter surveillance data (this includes data for patient episodes at other hospitals).

Results The study showed that over the 21 years, there has been a rapid growth in number of patients on HPN.

Number of patients on HPN by year.

The gender split of the cohort was 101 males and 120 females, with a total mean age 52.7 years and median age 55 years. Teesside $(22 \%, 49 / 221)$ followed by Northumberland $(18 \%, 40 / 221)$ were the regions with the most patients initiated on HPN.

The three most common indications for HPN were Postoperative complications (30.3\%, 67/221), Crohn's disease (21.3\%, 47/221) and Mesenteric ischaemia (16.7\%, 37/221). $14.9 \%(33 / 221)$ patients were on $\mathrm{HPN}$ as a result of malignancy.

The 1 year and 5 year survival for the cohort was 94\% and $79.4 \%$ respectively. There were 73 deaths noted in the study group, 8 patients had stopped HPN prior to death. 29 of these patients had been on HPN of a result of malignancy. Of the 65 patients who died while being on HPN, only four deaths were clearly as a complication of parenteral nutrition. 8 patients had neuroendocrine tumours; their average time on HPN was significantly greater at 25.6 months compared to 5.6 months for patients with other tumour types.

The mean rate of catheter-related bloodstream infection was 0.27 per 1000 catheter days over the period covering years 2011 till 2017. The rate of liver (significant fibrosis/cirrhosis) or biliary complications while being on HPN was 12\% (27/221) and the commonest complication was biliary obstruction/cholangitis $(33.3 \%, 9 / 27) .6$ patients of the cohort underwent successful small bowel transplantation and were able to stop HPN.

Conclusions The HPN service at the Freeman has noted a rapid growth in patient numbers, with survival rates comparable to or better than that reported in the literature. Mortality was linked to disease process rather than HPN complications and line infection rate was low.

\section{PWE-121 NUTRITIONAL ASSESSMENT AND VITAMIN DEFICIENCIES IN PATIENTS WITH NETS}

${ }^{1}$ Mark Reynolds*, ${ }^{2}$ Sheryl Lim, ${ }^{3}$ Aled Rees, ${ }^{2}$ Reyhan Chaudhry, ${ }^{1}$ Jennifer Blackhouse, ${ }^{1}$ Mohid S Khan. 'Department of Gastroenterology, Cardiff and Vale University Health Board, Cardiff, UK; ${ }^{2}$ Cardiff University, Cardiff, UK; ${ }^{3}$ Department of Endocrinology, Cardiff and Vale University Health Board, Cardiff, UK

\subsection{6/gutjnl-2018-BSGAbstracts.355}

Introduction Neuroendocrine tumours (NETs) have diverse natural history and clinical syndromes. As a result of the disease or related to management, patients may have altered gut or pancreatic function that can cause nutritional deficiencies. There is a lack of consistent evidence-based dietetic guidance for patients with NETs.

This study evaluated whether nutritional status and nutritional deficiencies had been assessed in patients with NETs in an existing service in South Wales.

Methods A retrospective study included 141 NET patients seen in Gastroenterology $(n=74)$ and Endocrinology $(n=67)$ clinics. Key parameters collected were: BMI, weight, vitamin B12, Ferritin, Folate, Albumin, vitamins $\mathrm{A} / \mathrm{D} / \mathrm{E}$ and presence of steatorrhoea. Evidence of treatment with vitamin or iron replacement and use of bile acid sequestrants or Creon was also recorded.

Results Weight was recorded in under half of patients $(70 / 141)$ and BMI in just $14 \%(n=20)$. This rose to $100 \%$ and $73 \%$ respectively in patients seen by a gastro-specialist dietitian; only 22 patients $(16 \%)$ had this specialist input. 54 patients reported weight loss, $70 \%$ of these $(n=38)$ had a quantified weight loss, $46 \%$ had percentage weight loss calculated.

106 patients $(75 \%)$ had been investigated for a form of vitamin or iron deficiency. The likelihood of investigation was significantly higher in Gastroenterology clinic patients than Endocrine clinics (95\% vs. $54 \%, \mathrm{p}<0.01$ ). $57 \%$ of those investigated were found to have a deficiency, which was consistent across specialities: 59\% $(41 / 70)$ of those from Gastroenterology clinics and 53\% (19/36) from Endocrine clinics. 41/60 patients (68\%) with a recorded deficiency did not have sufficient replacement. $7 / 27$ patients with iron deficiency were given supplementation. 38 patients had vitamin D levels tested (27\%), 29 were insufficient (76\%).

27 patients reported steatorrheoa, 26 of whom were prescribed somatostatin analogues. $96 \%$ of these patients were also prescribed Creon.

Conclusions Although higher rates of nutritional assessment were found in patients who had been assessed by Gastroenterology and with gastro-specialist dietetic involvement, assessment and management of nutritional status in patients with NETs remains an unmet need. Further evidence is required to evaluate nutritional assessment in NETs.

\section{PWE-122 NON-COELIAC GLUTEN/FRUCTAN SENSITIVITY OR IRRITABLE BOWEL SYNDROME (IBS)?}

${ }^{1}$ Kamran Rostami, ${ }^{1}$ Jafer Ali, ${ }^{2}$ Alison Parr, ${ }^{1}$ Ravi Madhotra, ${ }^{1}$ George Macfaul, ${ }^{3}$ Mohammad RostamiNejad. 'Department of Gastroenterology, Milton Keynes University Hospital, Milton Keynes, UK; ${ }^{2}$ Free Lance Nutrition Therapist, Manchester, UK; ${ }^{3}$ Gastroenterology and Liver Diseases Research Institute Shahid Beheshti University of Medical Sciences, Tehran, Iran

10.1136/gutjnl-2018-BSGAbstracts.356 